\title{
Article
}

\section{SATELLITE: Application to Planetary Nebulae IFU Data}

\author{
Stavros Akras ${ }^{1, * \mathbb{D}}$, Hektor Monteiro ${ }^{2} \mathbb{D}$, Jeremy R. Walsh ${ }^{3} \mathbb{D}$, Isabel Aleman ${ }^{2} \mathbb{D}$, Denise R. Gonçalves ${ }^{4}$ \\ and Panayotis Boumis ${ }^{1}$ (D)
}

1 Institute for Astronomy, Astrophysics, Space Application and Remote Sensing, National Observatory Athens, 15236 Athens, Greece; ptb@astro.noa.gr

2 Instituto de Física e Química, Universidade Federal de Itajubá, Pinheirinho, Itajubá 37500-903, Brazil; hektor.monteiro@gmail.com (H.M.); bebel.aleman@gmail.com (I.A.)

3 European Southern Observatory, 85748 Garching, Germany; jwalsh@eso.org

4 Observatório do Valongo, Universidade Federal do Rio de Janeiro, Rio de Janeiro 20080-090, Brazil; denise@astro.ufrj.br

* Correspondence: stavrosakras@gmail.com

Citation: Akras, S.; Monteiro H.; Walsh, J.R.; Aleman, I.; Gonçlves, D.R.; Panayotis, B. SATELLITE: Application to Planetary Nebulae IFU Data. Galaxies 2022, 10, 27. https://doi.org/10.3390/ galaxies10010027

Academic Editors: Martín Guerrero, Noam Soker and Quentin A. Parker

Received: 31 December 2021

Accepted: 26 January 2022

Published: 1 February 2022

Publisher's Note: MDPI stays neutral with regard to jurisdictional claims in published maps and institutional affiliations.

Copyright: () 2022 by the authors Licensee MDPI, Basel, Switzerland. This article is an open access article distributed under the terms and conditions of the Creative Commons Attribution (CC BY) license (https:// creativecommons.org/licenses/by/ $4.0 /)$.

\begin{abstract}
The integral field unit (IFU) spectroscopic view of extended ionized nebulae, such as planetary nebulae (PNe), H II regions, and galaxies, has changed the approach of studying these objects, providing a simultaneous characterization in both spatial directions. However, the spatial spaxel-by-spaxel analysis of such nebulae through IFUs is not directly comparable with the results obtained from the traditional slit-aperture spectroscopy or the predictions from 1D modelling. The new Python software called "SATELLITE: Spectroscopic Analysis Tool for intEgraL fieLd unIt daTacubEs" is used in the analysis of the VIMOS and MUSE datacubes of four Galactic PNe. The 2D analysis of line ratio maps has shown important variations from one to another nebular component in NGC 7009 and NGC 6778. In particular, the knots in both PNe are characterized by strong emission from neutral gas that is weak or even absent from the main nebula, indicating significant variation in the ionization state and density structure among the nebular components. The far-UV radiation from the central star results in the photo-evaporation of the dense molecular knots resembling the spectrum of photodissociation regions.
\end{abstract}

Keywords: integral field spectroscopy; optical spectroscopy; planetary nebulae; data analysis

\section{Introduction}

Planetary nebulae (PNe) are the descendants of low-to-intermediate mass stars (1-8 $\left.\mathrm{M}_{\odot}\right)$. The illumination of the material expelled during the asymptotic giant branch (AGB) stage by the exposed hot and luminous core causes the nebula to glow. The strong UV radiation field from their central stars (CS) is the dominant source of highly energetic photons, leading first to the dissociation of the cold AGB molecular gas and then to the ionization of the atomic gas.

PNe display a variety of shapes, such as round, elliptical, bipolar/multipolar, and point symmetric with different components, such as shells, rims, halos, knots, and filaments, among others. This polymorphism of PNe is attributed to complex post-AGB mass-loss events and/or mass transfer between components in a binary system, the presence or not of a dense equatorial structure (disk or torus), formation of jets and clumps, etc.

This structural complexity of PNe in conjunction with the UV radiation from the central stars results in an even more complex ionization and density stratification. Long-slit spectroscopic results are limited to specific directions or distinct components/features, such as low-ionization structures (LISs: knots, jets, and filaments; e.g., [1-3]) or bipolar outflows (e.g., [4-6]) due to the slit positioning.

The development of state-of-the-art spectrographs equipped with integral field units (IFU) overcome this limitation. Integral field spectroscopy (IFS) has changed the way we 
approach and study extended ionized nebulae, such as PNe, e.g., [7,8], H II regions [9], galaxies [10], and supernova remnants [11], simultaneously providing spectroscopic characterization in both spatial directions. However, the results from IFUs are not directly comparable with the results from the traditional long-slit spectroscopy or the predictions from 1D photoionization and shock modelling.

A new Python software called "SATELLITE: Spectroscopic Analysis Tool for Integral Field Init Datacubes"(Akras et al., 2021, submitted) has been developed to carry out a 1D and 2D analysis of extended nebulae. We present here a summary of some results from the application of the SATELLITE code to four Galactic PNe. A brief description of SATELLITE is given in Section 2. The results are presented in Section 3, and we finish with conclusions in Section 4.

\section{Methodology}

The SATELlite code (v1.3) was developed in Python 3, and four modules were available: (A) rotation analysis, (B) radial analysis, (C) specific slits analysis and (D) 2D analysis. A summary of the code is given below. A detailed description and discussion of its performance are presented in Akras et al., 2021 (submitted).

The 2D flux maps of emission lines extracted from the datacubes of any IFU are provided to the code as the input data ${ }^{1}$. The first three modules perform a 1D spectroscopic analysis through pseudo-slits or rectangular apertures spectra that simulate/resemble real long-slit spectra, summing up the values of the individual spaxels within the aperture defined by the width and length of the pseudo-slits that the user provides. The fourth module (D) carries out a 2D spectroscopic analysis through a spaxel-by-spaxel approach.

The following two criteria, (i) $\mathrm{F}(\mathrm{H} \alpha)>0$ and $\mathrm{F}(\mathrm{H} \beta)>0$ and (ii) $\mathrm{F}(\mathrm{H} \alpha)>2.85^{*} \mathrm{~F}(\mathrm{H} \beta)$, are taken into account to exclude problematic spaxels or those with low signal-to-noise $(\mathrm{S} / \mathrm{N}<2)$ ratio and avoid unrealistic values for the interstellar extinction coefficient $(\mathrm{c}(\mathrm{H} \beta))$.

The physical parameters of the nebula, such as $\mathrm{c}(\mathrm{H} \beta)$, line fluxes and intensities, electron temperatures $\left(T_{e}\right)$ and densities $\left(n_{e}\right)$ for all possible line diagnostics, ionic and total abundances, and ionization correction factors (ICFs), are computed using the PYNEB package $[12]^{2}$.

The current version of SATELLITE mimics the orientation of the pseudo-slits (position angle (PA)) by rotating the entire line flux maps instead of the slits themselves. Figure 1 shows the variation in $\mathrm{c}(\mathrm{H} \beta), T_{e}\left[\mathrm{~N}\right.$ II], and $n_{e}[\mathrm{~S}$ II] for NGC 7009 (upper panels), NGC 6778 (middle panels), and Hen 2-108 (lower panels) as a function of the PA of a rectangular aperture that covers the whole nebulae or, in other words, the entire line maps (integrated spectrum) of $64 \times 64$ arcsec, $67 \times 67$ arcsec, and $40 \times 40$ arcsec, respectively. The angular sizes of the PNe are: $56 \times 24 \operatorname{arcsec}$ NGC 7009, $60 \times 20 \operatorname{arcsec}$ NGC 6778, and 13-14 arcsec Hen 2-108, respectively.

The rotation of the flux maps does not have any impact on the integrated light across the nebulae. The SATELLITE benchmark tests verified, as expected, that all physical parameters (the ionic and elemental abundances and ionization correction factors (not shown here)) are invariant with the rotation of the input line flux maps. 

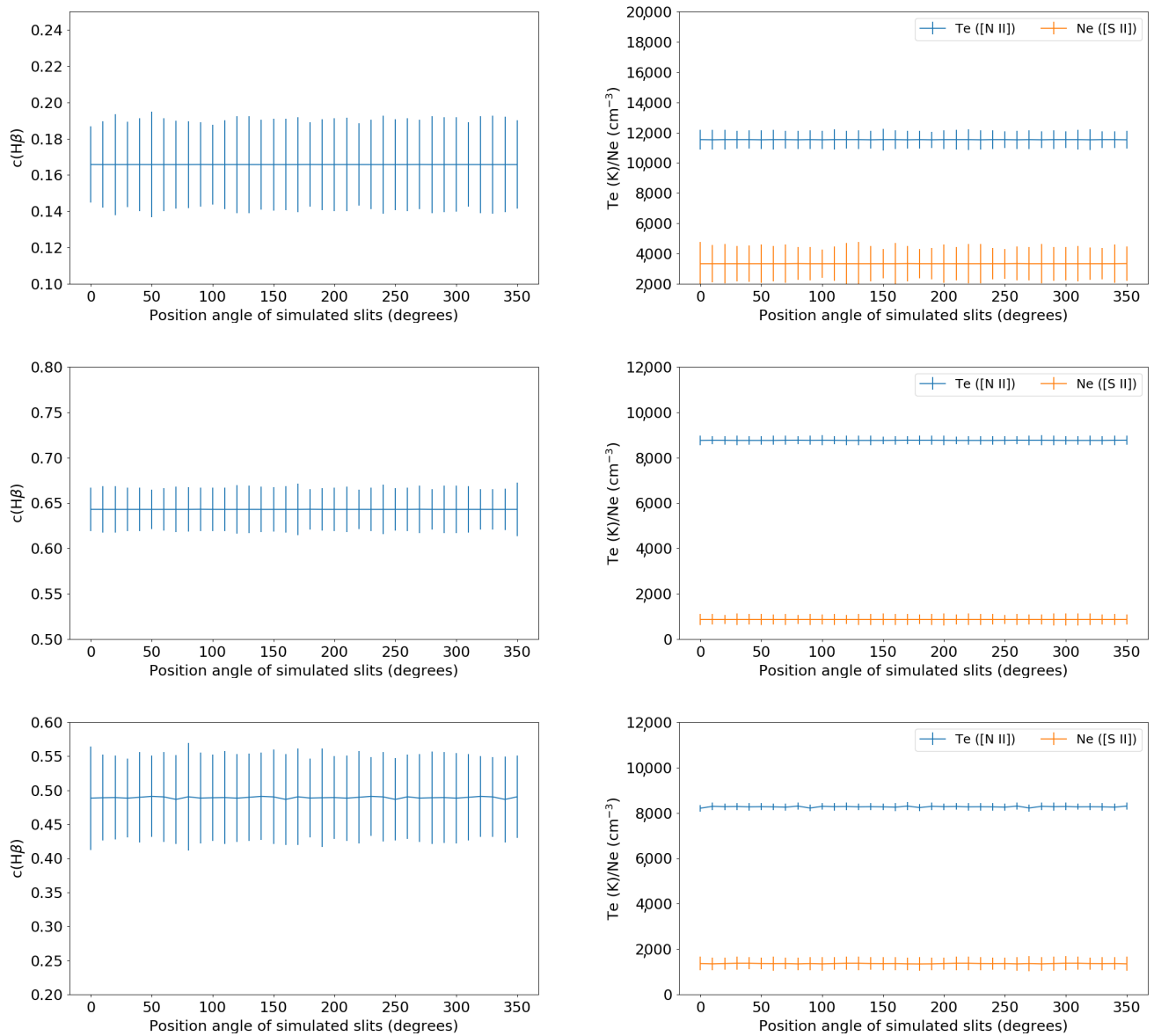

Figure 1. Interstellar extinction coefficient $\mathrm{c}(\mathrm{H} \beta)$ (left panels), electron temperature ( $T_{e}$, blue points), and density ( $n_{e}$, orange points) (right panels) of NGC 7009 (upper), NGC 6778 (middle), and Hen 2108 (lower panels) as functions of the position angle (PA) of the rectangular aperture that covers the whole nebulae $(64 \times 64 \mathrm{arcsec}, 67 \times 67 \mathrm{arcsec}, 40 \times 40 \mathrm{arcsec})$. The vertical lines indicate the error bars of each parameter.

\section{Results}

SATELLITE was used in the analysis of four Galactic PNe so far: Abell 14 (VIMOS, [7]), Hen 2-108 (VIMOS, Miranda-Marques et al., 2021 submitted), NGC 7009, and NGC 6778 (MUSE, Akras et al., 2021 submitted). Below, we briefly present some of the results obtained for each one.

\subsection{Abell 14}

The first (beta) version of SATELLITE was applied to the VIMOS@ESO [13] IFU data of the planetary nebula Abell 14 (PI: S. Akras; ID:098.D-0436(A)). Unexpectedly strong [N I] $\lambda 5200$ and [O I] $\lambda 6300$ emission lines were reported for this PN by $[14,15]$, respectively. The detection of only one of these two lines in each study above is likely related to the different slit positions ( 0 and $100{ }^{\circ} \mathrm{C}$, respectively). 3D photo-ionization modelling of Abell 14 has shown that the intensity of the aforementioned auroral lines cannot be reproduced considering only the UV radiation field from its CS, and an additional thermal mechanism is needed [16].

Therefore, Abell 14 was an ideal nebula to apply and test the SATELLITE code and in the end explore the line ratios in both spatial directions through the 2D analysis module, as well as for the specific slit orientations through the specific slits analysis module in the code (see, [7]). [N I] $\lambda 5200 / \mathrm{H} \beta$ ratio was found to be in agreement with the result reported by [14]. On the contrary, the [O I] $\lambda 6300 / \mathrm{H} \alpha$ line ratio was almost not measurable in 1D 
pseudo-slits at any PA. For the entire nebula (integrated spectrum), the measured ratio is half of the value reported by [15].

\subsection{Hen 2-108}

The first IFU data of Hen 2-108 are presented in Miranda-Marques et al. (2021, submitted), which describes the application of SATELLITE (v1.3) to the VLT/VIMOS data of this nebula (PI: H. Schwarz, ID:079.D-0117A).

Despite its nearly round shape, the radial analysis module in SATELLITE revealed a sharp drop in $\mathrm{c}(\mathrm{H} \beta)$ by $\sim 30 \%$ at a position angle of $\sim 50{ }^{\circ} \mathrm{C}$, while $T_{e}[\mathrm{~N} \mathrm{II}]$ and $n_{e}[\mathrm{~S}$ II $]$ are nearly constant with average values of $8230 \mathrm{~K}$ and $1390 \mathrm{~cm}^{-3}$ and standard deviations of $180 \mathrm{~K}$ and $215 \mathrm{~cm}^{-3}$, respectively. Homogeneity was also found for the ionic and total chemical abundances in this nebula.

The results from the radial analysis module also demonstrated that $\mathrm{c}(\mathrm{H} \beta)$ is lower in the inner part of the nebula and increases to 0.5 (towards eastern part) and 0.8 (towards southern part) at larger distances from the CS (Figure 2). Based on the 2D analysis module and a spaxel-by-spaxel approach, $\mathrm{c}(\mathrm{H} \beta), T_{e}[\mathrm{~N}$ II $]$, and $n_{e}[\mathrm{~S}$ II] vary in a wide range of values from 0.25 to 2, with a peak at 0.5 , from 5000 to $15,000 \mathrm{~K}$, with a peak at $8500 \mathrm{~K}$, and from 500 to $5000 \mathrm{~cm}^{-3}$, with a peak at $1000 \mathrm{~cm}^{-3}$.

The comparison of results between the 1D and 2D spectroscopic analyses illustrates the disparity of the two approaches due to the different $\mathrm{S} / \mathrm{N}$ ratios. Specifically, the S/N ratios of individual spaxels in the $2 \mathrm{D}$ analysis can be as low as 2 (at the outer part of the nebula) while the $\mathrm{S} / \mathrm{N}$ ratios are higher in the $1 \mathrm{D}$ analysis as emission from several spaxels is summed up. Note that the peak values from the 2D analysis agree with the results from the $1 \mathrm{D}$ analysis.

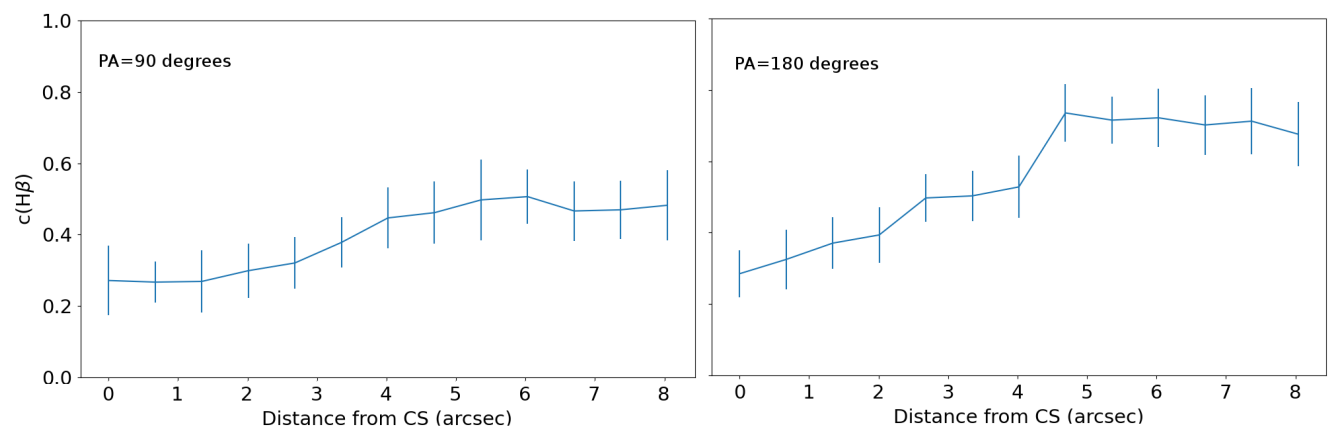

Figure 2. Radial profile of $\mathrm{c}(\mathrm{H} \beta)$ for Hen 2-108 along two pseudo-slits with PA of $90{ }^{\circ} \mathrm{C}$ (left panel) and $180^{\circ} \mathrm{C}$ (right panel).

\subsection{NGC 7009}

From a morphological point of view, NGC 7009 is among the richest nebulae, composed of an inner ellipsoidal structure, two pairs of knots (inner and outer), a pair of seeminglyjet structures, and an elliptical halo. The pairs of knots or LISs in this nebula $[17,18]$ ) are substantially brighter in the low-ionization lines (e.g., [N II] $\lambda \lambda 6548,6584$, [S II] $\lambda \lambda 6716,6731$, [O I] $\lambda \lambda 6300,6363,[\mathrm{~N} \mathrm{I}] \lambda 5200$, among others) than their surrounding gas (see also, [1,19]). Moreover, the recent detection of a $\mathrm{H}_{2} \mathrm{v}=1-02.12 \mu \mathrm{m} \mathrm{S}(1)$ ro-vibrational line emanating from the LISs in NGC 7009 [20] or generally in PNe [21,22] has shown a strong link between the emission from low-ionization lines and molecular hydrogen. Possible explanations to this link are either a low velocity shock interaction with the nebular shell or the photo-evaporation of dense molecular knots resembling the spectrum of mini-photodissociation regions (PDRs, see [23]).

Hence, spatially resolved line ratio maps are essential in order to study the structure of the nebula in both directions, as well as in different components. IFS is the ideal tool for such studies. NGC 7009 was observed with MUSE@ESO [24] during the Science Verification phase. The first results from this MUSE dataset are presented in $[25,26]$. The same set of emission line maps was used as input to SATELLITE to test and validate the code. The 
[S II] $\lambda 6716+\lambda 6731 / \mathrm{H} \alpha$, [O III] $\lambda 4959+\lambda 5007 / \mathrm{H} \beta$, [O I] $\lambda 6300 / \mathrm{H} \alpha$, and [N I] $\lambda 5200 / \mathrm{H} \beta$ line ratio maps of NGC 7009, corrected for interstellar extinction, obtained with SATELLITE are shown in Figure 3. The current version of SATELLITE provides 36 different combinations of emission line ratios.

The pairs of LISs are easily perceptible in the low-ionization line ratio maps with significantly higher values than the surrounding gas, whilst they also have lower [O III]/H $\beta$ values. Note that [O I] $\lambda 6300$ and [N I] $\lambda 5200$ line are mainly detected at the two pairs of knots, while they are absent or very weak in the main ellipsoidal nebula. This signifies that all $\mathrm{O}$ and $\mathrm{N}$ of the main nebula is ionized. The detection of these two low-ionization lines at the pair of knots indicates the presence of nebular components with distinct ionization states and density structures. This is consistent with the discovery of $\mathrm{H}_{2}$ gas, which requires high densities $\left(>10^{4-5} \mathrm{~cm}^{-3}\right)$ to be self-shielded from the dissociating radiation of the CS.
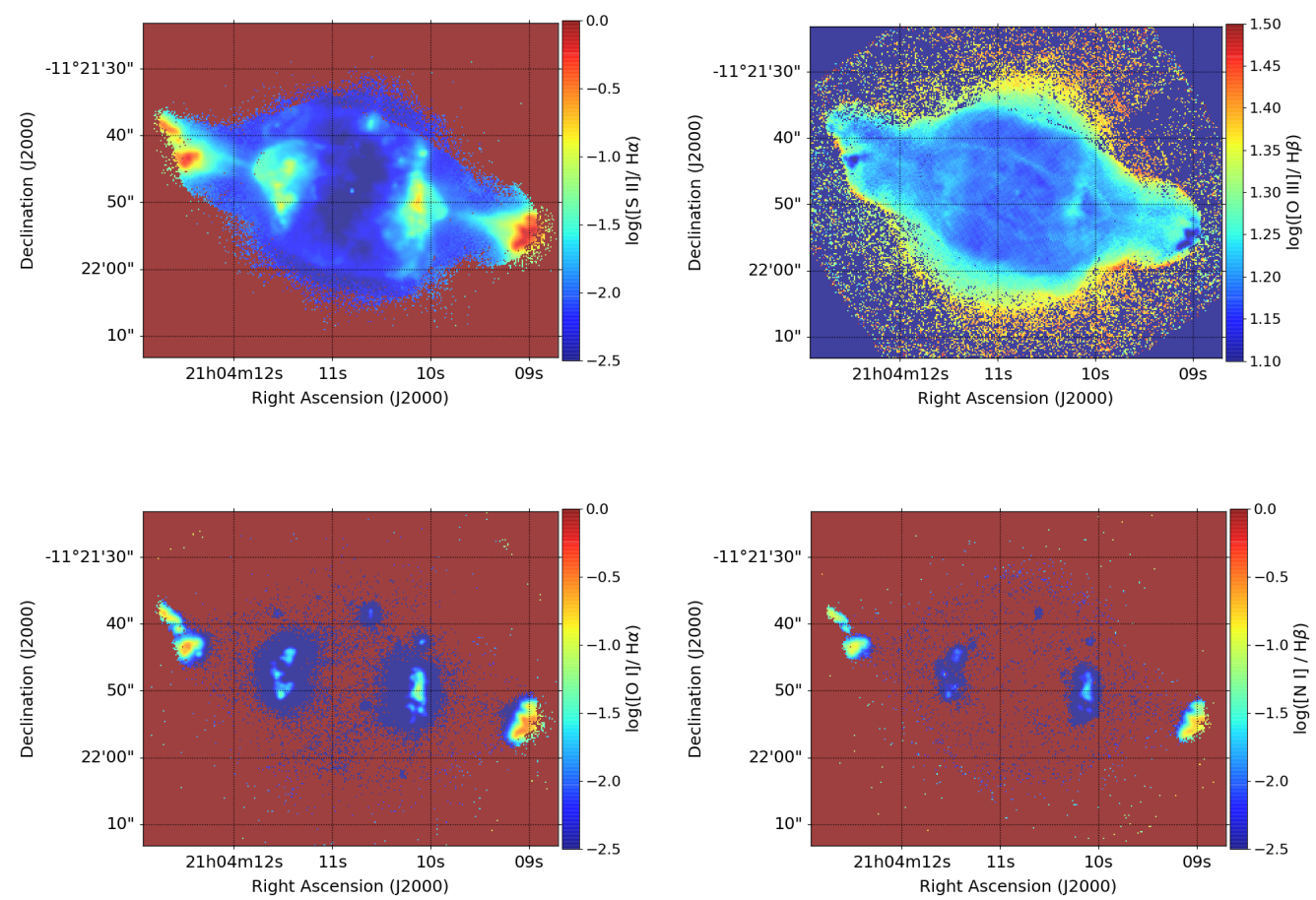

Figure 3. Emission line ratio maps of NGC 7009: [S II] $\lambda 6716+\lambda 6731 / \mathrm{H} \alpha$ (upper left panel), [O III] $\lambda 4959+\lambda 5007 / \mathrm{H} \beta$ (upper right panel), [O I] $\lambda 6300 / \mathrm{H} \alpha$ (lower left panel), and [N I] $\lambda 5200 / \mathrm{H} \beta$ (lower right panel).

Moreover, a clear stratification in the knots was also observed. Both line ratios, [O I] $\lambda 6300 / \mathrm{H} \alpha$ and $[\mathrm{N} \mathrm{I}] \lambda 5200 / \mathrm{H} \beta$, had low values $(>-2.5)$ closer to the CS and increased to higher distances or deeper into the knots up to -0.5 (see Figure 4 ). On the other hand, the [O III] $/ \mathrm{H} \beta$ line ratio was higher at the outer parts of the knots and became lower deeper in the structures or at a higher distance from the CS (not shown here). This stratification in the knots is also verified by the radial analysis module, which provides the peak of each emission along the radial direction. This result agrees with the prediction from the photo-evaporation of dense molecular clumps [23].

The outer edges of the nebula are characterized by the highest [O III] $/ \mathrm{H} \beta$ ratio $>1.35$ (upper right panel in Figure 3). This augmentation in the [O III]/ $\mathrm{H} \beta$ ratio was also found in other PNe and attributed to potential shock interactions with the ISM [4].

\subsection{NGC 6778}

NGC 6778 displays a complex morphology characterized by a bright waist and a number of collimated outflows and knots [27]. SATELLITE was applied to the MUSE IFU data of this nebula (PI: R. L. M. Corradi; ID: 097.D-0241(A)) to carry out a 1D and 2D 
spectroscopic studies. A more detailed analysis of the nebula, including several optical recombination lines (ORLs), is presented by [8].
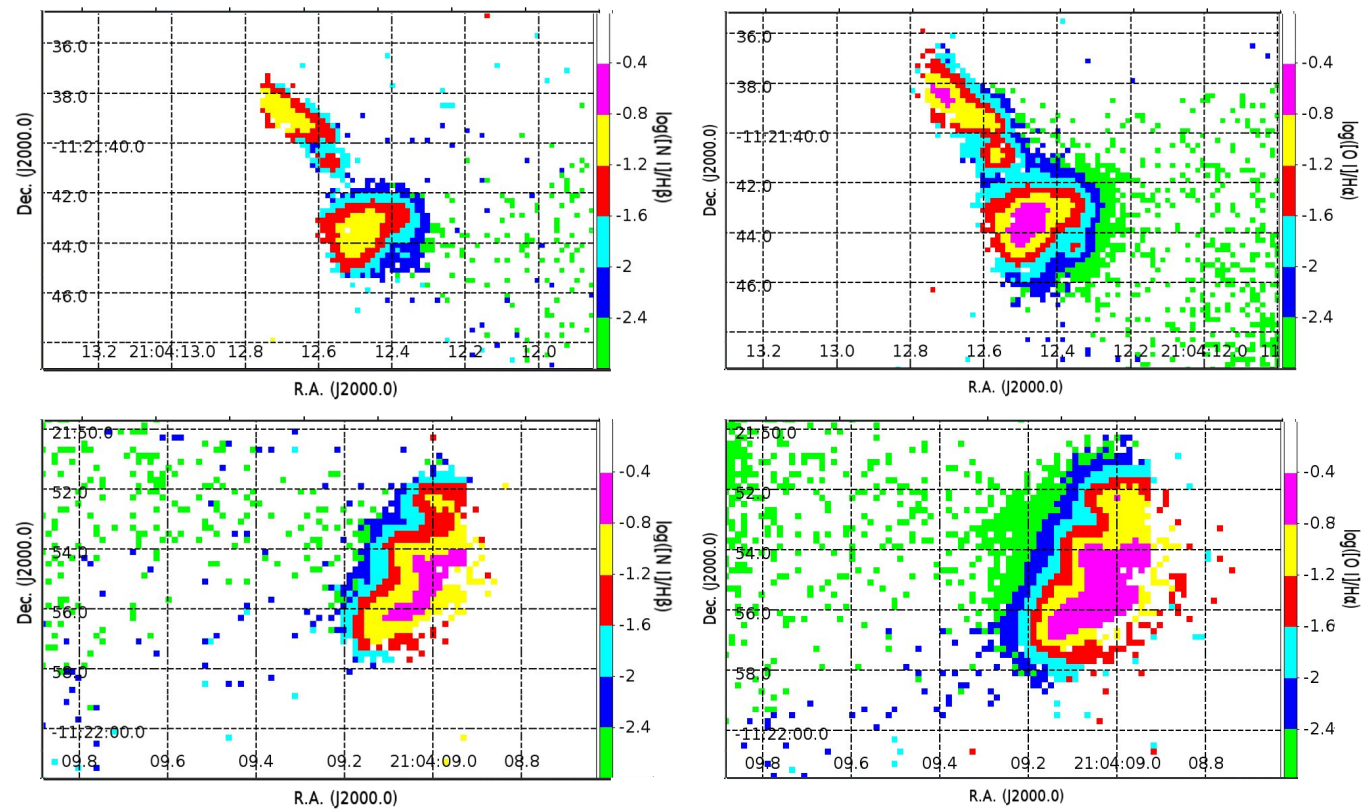

Figure 4. $\log \left(\left[\mathrm{N}_{\mathrm{I}}\right] \lambda 5200 / \mathrm{H} \beta\right)$ (left panels) and $\log ([\mathrm{O} \mathrm{I}] \lambda 6300 / \mathrm{H} \alpha)$ (right panels) line ratios of the northeast (upper panels) and southwest (lower panels) LISs in NGC 7009.

Figure 5 displays the [S II $\lambda 6716+\lambda 6731 / \mathrm{H} \alpha$, [O III $\lambda 4959+\lambda 5007 / \mathrm{H} \beta$, [O I] $\lambda 6300 / \mathrm{H} \alpha$, and $[\mathrm{NI}] \lambda 5200 / \mathrm{H} \beta$ line ratio maps of NGC 6778. The waist of the nebula seems to be fragmented into several filaments and knots. All these micro-structures exhibit higher low-ionization line ratios, as well as higher [O III] $/ \mathrm{H} \beta$ ratio relative to the surrounding gas, contrary to the outer isolated knots at the edges of the collimated outflows, which are characterized by higher low-ionization line ratios and lower [O III]/ $\mathrm{H} \beta$ ratio. The same hypothesis of nebular components with different ionization states and density structures is also applicable to NGC 6778.
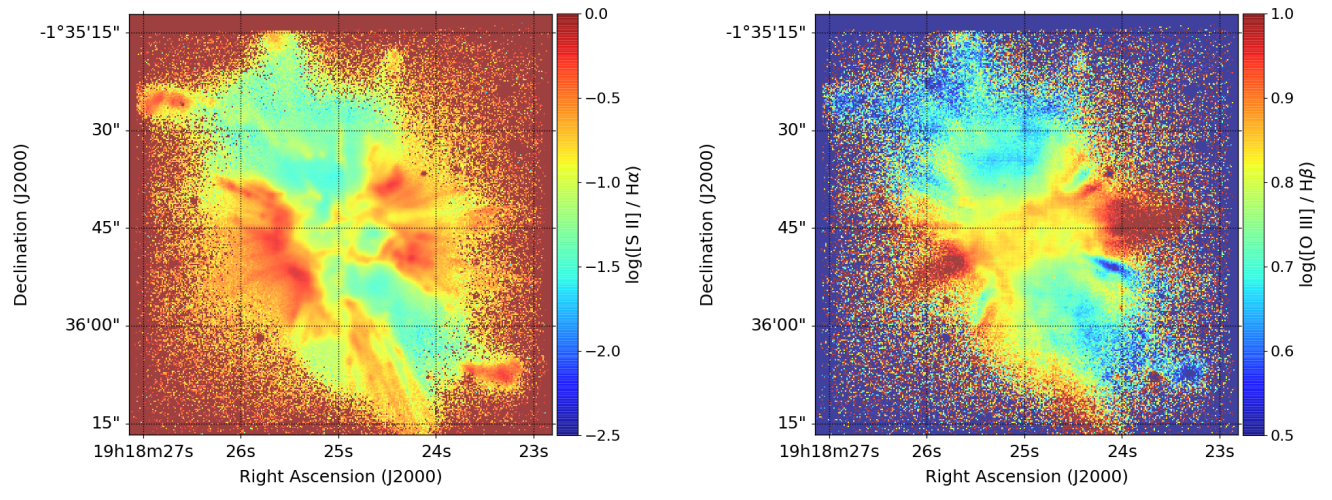

Figure 5. Cont. 

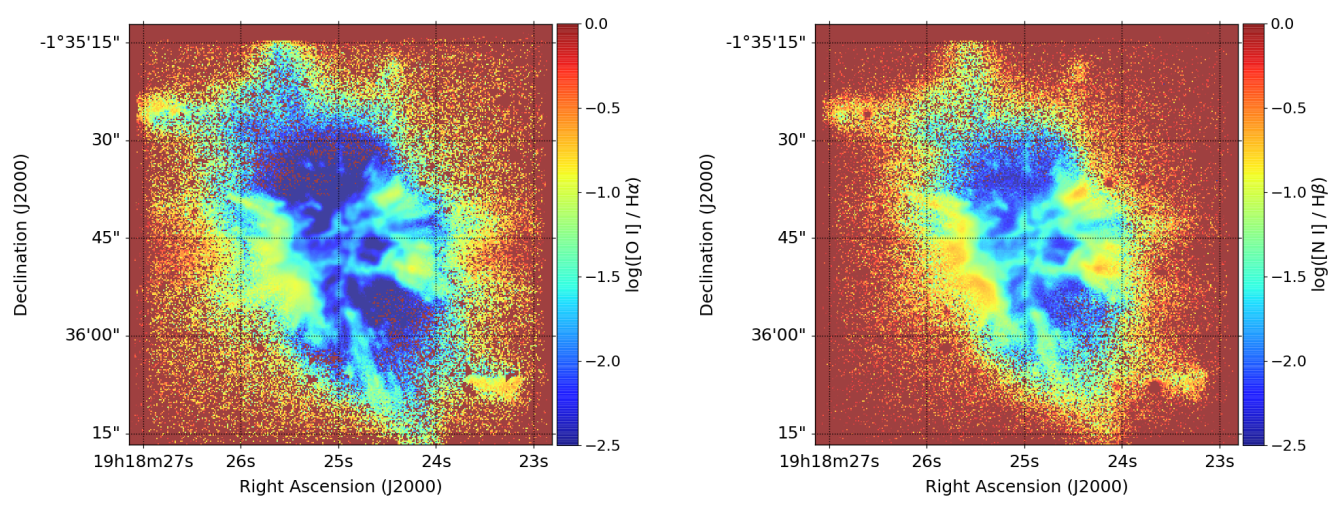

Figure 5. Emission line ratio maps of NGC 6778: [S II] $\lambda 6716+\lambda 6731 / \mathrm{H} \alpha$ (upper left panel), [O III] $\lambda 4959+\lambda 5007 / \mathrm{H} \beta$ (upper right panel), [O I] $\lambda 6300 / \mathrm{H} \alpha$ (lower left panel), and [N I] $\lambda 5200 / \mathrm{H} \beta$ (lower right panel).

\section{Conclusions}

Integral field spectroscopy has changed the way we study extended ionized nebulae, such as PNe galaxies or H II regions. However, the comparison between the spaxel-by-spaxel analysis of IFU and the traditional long-slit spectroscopy or 1D modelling is not straightforward $[7,28]$. A new Python code, namely SATELLITE, was recently developed (Akras et al. 2021, submitted) to carry out 1D (pseudo-slits) and 2D (spaxel-by-spaxel) spectroscopic analyses of the line maps provide by IFUs.

The results from the application of SATELLITE to the VIMOS and MUSE IFU data of four PNe are discussed in this contribution and are summarised below.

- The orientation or position angle of the pseudo-slits in SATELLITE is simulated by rotating the entire line flux maps. This rotation has no impact on the calculations of any physico-chemical parameter.

- The 1D spectroscopic analysis of the VIMOS datacubes of Abell 14 through the specific slit module has confirmed the high [N I] $\lambda 5200 / \mathrm{H} \beta$ ratio reported in the literature, but not the high [O I] $\lambda 6300 / \mathrm{H} \alpha$ ratio, which is not measurable in the $1 \mathrm{D}$ pseudo-slits from any PA.

- Hen 2-108 is characterized by a low $\mathrm{c}(\mathrm{H} \beta)$ in the inner part of the nebula that increases towards the outer parts based on the results from the radial analysis module. Interestingly, the rotation analysis module has also revealed a non-negligible reduction of $\mathrm{c}(\mathrm{H} \beta)$ at the position angle of $\sim 50^{\circ} \mathrm{C}$.

- The analysis of the 2D emission line ratio maps of NGC 7009 has displayed a significantly different ionization state among the different nebular features. Neutral gas is found mainly in the pairs of knots.

- $\quad$ NGC 6778 shows a knotty and filamentary waist, together with some knots at the edges of the collimated outflows. All knots have $\log ([\mathrm{N} \mathrm{I}] \lambda 5200 / \mathrm{H} \beta)>-1$ and $\log ([\mathrm{O} \mathrm{I}]$ $\lambda 6300 / \mathrm{H} \alpha)>-1$, while the main nebula exhibits ratios $<-2$.

- The variation in the ionization state between the knots and the main nebular components may indicate structures with significant density contrast. The photo-evaporation process is likely responsible for the emission from the dense molecular knots.

The use of SATELLITE makes the quantitative evaluation of the physic-chemical $(\mathrm{c}(\mathrm{H} \beta)$, $T_{e}, N_{e}, \mathrm{X} / \mathrm{H}$, etc) variations far easier, giving the extended nebulae a great opportunity to dive in on the exploration and interpretation of these variation behaviours for a more complete understanding of these ionized nebulae. The code itself will also be upgraded by improving its capabilities and implementing additional modules and tasks.

Author Contributions: Methodology, software, validation, S.A.; writing—original draft preparation, S.A., H.M., J.R.W., I.A., D.R.G. and P.B.; writing-review and editing, S.A., H.M., J.R.W., I.A., D.R.G. and P.B. All authors have read and agreed to the published version of the manuscript. 
Funding: S.A. acknowledges support under the grant 5077 (The EXPANSE project), financed by IAASARS/NOA. D.R.G. is partially supported by the CNPq grants 428330/2018-5 and 313016/2020-8

Data Availability Statement: MUSE and VIMOS data presented in this work are available at the ESO online Archive database (http:/ / archive.eso.org/cms.html) (accessed on 23 November 2021).The SATELLITE code, along with the documentation and examples, is available at the GitHub repository (https: / / github.com/StavrosAkras/SATELLITE.git) (accessed on 23 November 2021).

Conflicts of Interest: The authors declare no conflict of interest.

\begin{tabular}{|c|c|}
\hline \multicolumn{2}{|c|}{ Abbreviations } \\
\hline T & ing abbreviations are used in this \\
\hline PN & Planetary Nebula \\
\hline AGB & Asymptotic giant branch \\
\hline IFU & Integral Field Unit \\
\hline MUSE & Multi-Unit Spectroscopic Explorer \\
\hline VIMOS & VIsible MultiObject Spectrograph \\
\hline ESO & European Southern Observatory \\
\hline ORLs & Optical Recombination Lines \\
\hline IFS & Integral field spectroscopy \\
\hline PDRs & Photodissociation regions \\
\hline ICF & Ionization correction factor \\
\hline LISs & Low ionization structures \\
\hline PA & Position angle \\
\hline CS & Central star \\
\hline
\end{tabular}

\section{Notes}

SATELLITE does not extract the 2D flux maps from IFU data cubes. These maps are provided by the user and they can be derived either by the spectral fitting of an emission line (this is the case for all four PNe presented in this work) or summing up the flux density over a given wavelength range.

2 The PYNEB versions 1.1.13 and 1.1.15 were tested. The latest version of PYNEB was implemented in the current version of SATELLITE (v1.3).

\section{References}

1. Akras, S.; Gonçalves, D.R. Low-ionization structures in planetary nebulae - I. Physical, kinematic and excitation properties. Mon. Not. R. Astron. Soc. 2016, 455, 930-961. [CrossRef]

2. Derlopa, S.; Akras, S.; Boumis, P.; Steffen, W. High-velocity string of knots in the outburst of the planetary nebula Hb4. Mon. Not. R. Astron. Soc. 2019, 484, 3746-3754. [CrossRef]

3. Balick, B.; Perinotto, M.; Maccioni, A.; Terzian, Y.; Hajian, A. FLIERs and Other Microstructures in Planetary Nebulae. II. Astrophys. J. 2015, 424, 800. [CrossRef]

4. Guerrero, M.A.; Toalá, J.A.; Medina, J.J.; Luridiana, V.; Miranda, L.F.; Riera, A.;Velázquez, P.F. Unveiling shocks in planetary nebulae. Astron. Astrophys. 2015, 557, A121. [CrossRef]

5. Akras, S.; Boumis, P.; Meaburn, J.; Alikakos, J.; López, J.A.; Gonçalves, D.R. Evidence for a [WR] or WEL-type binary nucleus in the bipolar planetary nebula Vy 1-2. Mon. Not. R. Astron. Soc. 2015, 452, 2911-2929. [CrossRef]

6. Ramos-Larios, G.; Guerrero, M.A.; Nigoche-Netro, A.; Olguín, L.; Gómez-Muñoz, M.A.; Sabin, L.; Vázquez, R.; Akras, S.; Ramírez Vélez, J.C.; Chávez, M. The interaction of the halo around the butterfly planetary nebula NGC 650-1 with the interstellar medium. Mon. Not. R. Astron. Soc. 2018, 475, 932-941. [CrossRef]

7. Akras, S.; Monteiro, H.; Aleman, I.; Farias, M.A.F.; May, D.; Pereira, C.B. Exploring the differences of integrated and spatially resolved analysis using integral field unit data: The case of Abell 14. Mon. Not. R. Astron. Soc. 2020, 493, 2238-2252. [CrossRef]

8. García-Rojas, J.; Morisset, C.; Jones, D.; Wesson, R.; Boffin, H.M.J.; Monteiro, H.; Corradi, R.L.M.; Rodríguez-Gil, P. MUSE spectroscopy of planetary nebulae with high abundance discrepancies. Mon. Not. R. Astron. Soc. 2022, 510, 5444-5463. [CrossRef]

9. Kumari, N.; James, B.L.; Irwin, M.J. A GMOS-N IFU study of the central H II region in the blue compact dwarf galaxy NGC 4449: Kinematics, nebular metallicity and star formation. Mon. Not. R. Astron. Soc. 2017, 470, 4618-4637. [CrossRef]

10. Telles, E.; Thuan, T.X.; Izotov, Y.I.; Carrasco, E.R. A Gemini/GMOS study of the physical conditions and kinematics of the blue compact dwarf galaxy Mrk 996. Astron. Astrophys. 2014, 561, A64. [CrossRef]

11. Seitenzahl, I.R.; Vogt, F.P.A.; Terry, J.P.; Ghavamian, P.; Dopita, M.A.; Ruiter, A.J.; Sukhbold, T. Integral Field Spectroscopy of Supernova Remnant 1E0102-7219 Reveals Fast-moving Hydrogen and Sulfur-rich Ejecta. Astrophys. J. 2018, 853, L32. [CrossRef] 
12. Luridiana, V.; Morisset, C.; Shaw, R.A. PyNeb: A new tool for analyzing emission lines. I. Code description and validation of results. Astron. Astrophys. 2015, 573, A42. [CrossRef]

13. Le Fèvre, O.; Saisse, M.; Mancini, D.; Brau-Nogue, S.; Caputi, O.; Castinel, L.; D'Odorico, S.; Garilli, B.; Kissler-Patig, M.; Lucuix, C.; et al. Commissioning and performances of the VLT-VIMOS instrument. In Proceedings of the Astronomical Telescopes and Instrumentation, Waikoloa, HI, USA, 22-28 August 2002; Volume 4841, pp. 1670-1681.

14. Bohigas, J. Infrared Imaging and Optical Imaging and Spectroscopy of (mostly) Type I Planetary Nebulae. II. Rev. Mex. Astron. Astrofis. 2003, 39, 149-170.

15. Henry, R.B.C.; Kwitter, K.B.; Jaskot, A.E.; Balick, B.; Morrison, M.A.; Milingo, J.B. Abundances of Galactic Anticenter Planetary Nebulae and the Oxygen Abundance Gradient in the Galactic Disk. Astrophys. J. 2010, 724, 748-761. [CrossRef]

16. Akras, S.; Clyne, N.; Boumis, P.; Monteiro, H.; Gonçalves, D.R.; Redman, M.P.; Williams, S. Deciphering the bipolar planetary nebula Abell 14 with 3D ionization and morphological studies. Mon. Not. R. Astron. Soc. 2016, 457, 3409-3419. [CrossRef]

17. Gonçalves, D.R.; Corradi, R.L.M.; Mampaso, A.; Perinotto, M. The Physical Parameters, Excitation, and Chemistry of the Rim, Jets, and Knots of the Planetary Nebula NGC 7009. Astrophys. J. 2003, 597, 975-985. [CrossRef]

18. Gonçalves, D.R.; Ercolano, B.; Carnero, A.; Mampaso, A.; Corradi, R.L.M. On the nitrogen abundance of fast, low-ionization emission regions: The outer knots of the planetary nebula NGC 7009. Mon. Not. R. Astron. Soc. 2006, 365, 1039-1049. [CrossRef]

19. Gonçalves, D.R.; Corradi, R.L.M.; Mampaso, A. Low-Ionization Structures in Planetary Nebulae: Confronting Models with Observations. Astrophys. J. 2001, 547, 302-310. [CrossRef]

20. Akras, S.; Gonçalves, D.R.; Ramos-Larios, G.; Aleman, I. $\mathrm{H}_{2}$ emission in the low-ionization structures of the planetary nebulae NGC 7009 and NGC 6543. Mon. Not. R. Astron. Soc. 2020, 493, 3800-3810. [CrossRef]

21. Akras, S.; Gonçalves, D.R.; Ramos-Larios, G. $\mathrm{H}_{2}$ in low-ionization structures of planetary nebulae. Mon. Not. R. Astron. Soc. 2017, 465, 1289-1296. [CrossRef]

22. Fang, X.; Zhang, Y.; Kwok, S.; Hsia, C.-H.; Chau, W.; Ramos-Larios, G.; Guerrero, M.A. Extended Structures of Planetary Nebulae Detected in $\mathrm{H}_{2}$ Emission. Astrophys. J. 2017, 859, 92. [CrossRef]

23. Mellema, G.; Raga, A.C.; Canto, J.; Lundqvist, P.; Balick, B.; Steffen, W.; Noriega-Crespo, A. Photo-evaporation of clumps in planetary nebulae. Astron. Astrophys. 1998, 331, 335-346.

24. Bacon, R.; Accardo, M.; Adjali, L.; Anwand, H.; Bauer, S.; Biswas, I.; Blaizot, J.; Boudon, D.; Brau-Nogue, S.; Brinchmann, J.; et al. The MUSE second-generation VLT instrument. Ground-based and Airborne Instrumentation for Astronomy III. Proc. SPIE 2010, $7735,773508$.

25. Walsh, J.R.; Monreal-Ibero, A.; Barlow, M.J.; Ueta, T.; Wesson, R.; Zijlstra, A.A. The extinction and dust-to-gas structure of the planetary nebula NGC 7009 observed with MUSE. Astron. Astrophys. 2016, 588, A106. [CrossRef]

26. Walsh, J.R.; Monreal-Ibero, A.; Barlow, M.J.; Ueta, T.; Wesson, R.; Zijlstra, A.A. An imaging spectroscopic survey of the planetary nebula NGC 7009 with MUSE. Astron. Astrophys. 2018, 620, A169. [CrossRef]

27. Guerrero, M.A.; Miranda, L.F. NGC 6778: A disrupted planetary nebula around a binary central star. Astron. Astrophys. 2012, 539, A47. [CrossRef]

28. Ercolano, B.; Dale, J.E.; Gritschneder, M.; Westmoquette, M. Synthetic observations of simulated Pillars of Creation. Mon. Not. R. Astron. Soc. 2020, 420, 141-146. [CrossRef] 